\title{
Revista \\ GLI ACQUISTI ECO-SOSTENIBILI NEL NUOVO CODICE DEI CONTRATTI PUBBLICI ITALIANO: CONSIDERAZIONI SUL LIFE CYCLE COSTING
}

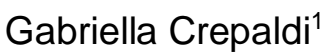

Recebido em: 03/05/2018

Aceito em: 02/06/2018

gabriella.crepaldi@unibg.it

\section{L'INQUADRAMENTO NORMATIVO DEGLI APPALTI VERDI IN ITALIA}

Gli acquisti pubblici in Italia rappresentano circa il 17\% del Prodotto Interno Lordo e nei paesi dell'Unione Europea mediamente il 14\%, con massimi che raggiungono il $25 \%$ nell'area scandinava: è proprio la mole della domanda che il settore pubblico rivolge al mercato che supporta l'affermazione secondo cui l'acquisto pubblico eco-sostenibile costituisce uno strumento di politica economica ambientale, come tale in grado di condizionare il comportamento degli operatori privati sul mercato²: se il pubblico richiede prodotti e servizi ecologici, gli operatori commerciali privati devono adeguare la propria offerta, se necessario investendo nella studio delle risorse, dei materiali, delle metodologie al fine del conseguimento di soluzioni produttive, di processo o di prodotto, ecocompatibili³.

Anche per la necessità di recuperare le spese sostenute per la ricerca e l'innovazione, i beni (al pari dei servizi e delle opere) compatibili con l'ambiente sono normalmente più costosi rispetto ai beni prodotti secondo le metodologie tradizionali: perciò, in sede di gara, ai primi non possono che corrispondere offerte da parte dellimpresa privata meno convenienti dal punto di vista strettamente economico rispetto a quelle calcolate senza badare all'eco-sostenibilità di ciò che si offre.

A ciò soccorre la disciplina normativa che, tanto nella formulazione del Codice dei contratti pubblici del 2006 (d.Igs. 12 aprile 2006, n. 163) in recepimento delle Direttive europee 17/2004/CE e 18/2004/CE, tanto quella dell'attuale Codice (d.Igs. 18 aprile 2016, n. 50) in attuazione delle Direttive 2014/23/UE, 2014/24/UE e 2014/25/UE ${ }^{4}$ prevede che "il principio di economicità può essere subordinato, nei limiti in cui è espressamente consentito dalle norme vigenti e dal presente codice, ai criteri, previsti nel bando, ispirati a esigenze sociali, nonché alla tutela della salute, dell'ambiente, del patrimonio culturale e alla promozione dello sviluppo sostenibile, anche dal punto di vista energetico".

\footnotetext{
${ }^{1}$ Università degli Studi di Bergamo - UniBG - Bérgamo - Itália

2 Si rinvia a M. CLARICH, La tutela dell'ambiente attraverso il mercato, in Dir. pubbl., 2007, 219 ss.; nonché in M. CAFAGNO, F. FONDERICO, Riflessione economica e modelli di azione amministrativa a tutela dell'ambiente, in P. DELL'ANNO, E. PICOZZA (diretto da), Trattato di diritto dell'ambiente, vol. I, Principi generali, Cedam, Padova, 2012, 487 ss.

${ }^{3}$ Sul punto v. la Comunicazione interpretativa della Commissione europea "Il diritto comunitario degli appalti pubblici e le possibilità di integrare considerazioni di carattere ambientale negli appalti pubblici" (COM/2001/274 def.).

${ }^{4}$ Per una panoramica sulle novità introdotte dalle Direttive europee del 2014 si rinvia a L. TORCHIA, La nuova direttiva europea in materia di appalti servizi e forniture nei settori ordinari, in Dir. amm., 2015, 291 ss.
} 
In quest'affermazione generale e in molte altre più specifiche ${ }^{5}$ si ravvisa uno scopo che va al di là di quello meramente economico e che attribuisce così alla regolazione della contrattualistica pubblica un diverso fine, coerente con gli obiettivi dell'Unione europea quale comunità non esclusivamente economica, bensì politica e sociale. Ecco allora che l'obiettivo prioritario della crescita economica contiene già in sé istanze ambientali e sociali che sono funzionali alla garanzia dello sviluppo sostenibile quale principio fondamentale della tutela ambientale ${ }^{6}$. In questo modo, il settore dei contratti pubblici viene ad integrarsi nelle politiche generali dell'Unione europea per lo sviluppo di un'economia ispirata all'innovazione e all'eco-sostenibilità'.

Con questa chiave di lettura, che segna la ratio della normativa nel suo complesso, devono essere studiate, interpretate ed applicate tutte le previsioni che introducono, nei vari momenti del procedimento di acquisto pubblico, la considerazione degli aspetti ambientali a partire da quelle che affermano i principi (v. art. 30) sino a quelle di dettaglio.

Si noti allora che il Codice del 2016, perfezionando quello le precedenti disposizioni del Codice del 2006, prevede vari momenti della procedura ad evidenza pubblica in cui viene ad assumere rilevanza la considerazione dell'interesse ambientale attraverso la valutazione delle imprese partecipanti alla gara e della prestazione offerta: i criteri ambientali, infatti, possono essere determinati tanto nell'ambito dei requisiti soggettivi per l'ammissione alla gara ${ }^{8}$ e della determinazione delle caratteristiche dell'oggetto del contratto $^{9}$, quanto come parametri per l'aggiudicazione dell'appalto, ovvero, infine, vincolando l'impresa vincitrice al rispetto di particolari clausole di esecuzione del contratto ${ }^{10}$.

\footnotetext{
${ }^{5}$ Si vedano, fra le altre, l'art. 38 (spec. comma IV, lett. b) n. 5, sulla qualificazione delle stazioni appaltanti; l'art. 68 , comma V, lett. a) sulle specifiche tecniche; l'art. 69 che regola le etichettature come mezzo di prova che i lavori, le forniture o i servizi corrispondono alle caratteristiche ambientali e sociali richieste; l'art. 76, comma II, in merito ai sistemi di gestione ambientale; l'art. 100, comma I, sulle condizioni di esecuzione del contratto.

${ }^{6}$ Sull'implementazione in Italia del principio dello sviluppo sostenibile si rinvia a C. VIDETTA, Lo sviluppo sostenibile. Dal diritto internazionale al diritto interno, in AA.VV., Trattato di diritto dell'ambiente, Milano, Giuffré, 2014, 221 ss.

${ }^{7}$ Si tenga conto della Strategia Europa 2020. Una strategia per una crescita intelligente, sostenibile e inclusiva che è stata oggetto della comunicazione della Commissione europea ( $\operatorname{COM}(2010) 2020)$ allo scopo di individuare tre priorità che si rafforzano a vicenda: crescita intelligente (sviluppare un'economia basata sulla conoscenza e sull'innovazione); crescita sostenibile (promuovere un'economia più efficiente sotto il profilo delle risorse, più verde e più competitiva); crescita inclusiva (promuovere un'economia con un alto tasso di occupazione che favorisca la coesione sociale e territoriale). Come ha evidenziato la Commissione europea, $\mathrm{i}$ criteri di aggiudicazione giocano un ruolo fondamentale nel perseguimento degli obiettivi della Strategia Europa 2020, in quanto “l'impiego di criteri associati all'ambiente, all'efficienza energetica, all'accessibilità o all'innovazione nella fase di aggiudicazione piuttosto che soltanto nelle specifiche tecniche o come condizioni di esecuzione dell'appalto può avere il vantaggio di spingere le aziende a presentare offerte che vanno al di là dei livelli fissati nelle specifiche tecniche e quindi promuovere l'introduzione di prodotti innovativi sul mercato" (Comunicazione sulla modernizzazione della politica dell'UE in materia di appalti pubblici. Per una maggiore efficienza del mercato europeo degli appalti - $\operatorname{COM(2011)15~def.).~}$

${ }^{8}$ Ciò in termini positivi, individuando requisiti di compatibilità ambientale di cui le imprese devono dimostrare il possesso, e in termini negativi, escludendo le imprese che abbiano commesso reati ambientali incidenti sulla moralità professionale.

9 In questo senso si vedano le disposizioni sulle "specifiche tecniche" (art. 68) e le "etichettature" (art. 69).

${ }^{10}$ Sia consentito di rinviare a G. CREPALDI, Politiche ambientali nei contratti pubblici degli enti locali, in M. ANDREIS (a cura di), I contratti pubblici tra principi interni e vincoli sopranazionali, Milano, Giuffré, 2011,195 SS.
} 
Operando nel solco tracciato dalla normativa previgente, il Codice dei contratti pubblici riconosce un ruolo fondamentale ai CAM (criteri ambientali minimi) stabiliti con l'adozione del Piano d'azione per la sostenibilità ambientale dei consumi nel settore della pubblica amministrazione (PAN GPP) di cui al decreto interministeriale 11 aprile 2008 n. 135 e al decreto interministeriale di aggiornamento del 10 aprile 2013.

II PAN individua dei settori merceologici di intervento prioritario degli acquisti verdi (fra i quali, gli arredi, l'edilizia, la gestione rifiuti, i servizi energetici, i servizi urbani, l'elettronica, la cancelleria, la ristorazione, i trasporti) e per ciascuno di essi rinvia alla definizione di criteri ambientali minimi da parte di successivi decreti del Ministero dell'Ambiente e della tutela del Territorio e del Mare (così, fra gli altri, il d.m. 25 luglio 2011 ha adottato i criteri ambientali minimi per l'acquisto da parte delle p.a. di prodotti e servizi nei settori della ristorazione; d.m. 4 aprile 2013 per acquisto di carta per copia e carta grafica; d.m. 24 maggio 2012 per l'affidamento del servizio di pulizia e per la fornitura di prodotti per l'igiene; d.m. 8 maggio 2012 per acquisizione dei veicoli adibiti al trasporto su strada), quali indicazioni tecniche, generali e specifiche, di natura prevalentemente ambientale che le pubbliche amministrazione devono considerare in sede di definizione delle specifiche della prestazione e di valutazione dell'offerta più vantaggiosa ${ }^{11}$.

L'art. 34 del Codice del 2016 come modificato dal Correttivo del 2017, in merito alla sostenibilità energetica ed ambientale, richiede l'inserimento nella documentazione progettuale e di gara "almeno delle specifiche tecniche e delle clausole contrattuali" contenute nei CAM: si tratta di un obbligo applicabile agli affidamenti di qualunque importo, relativamente alle categorie di forniture e di affidamenti di servizi e lavori oggetto dei criteri ambientali minimi adottati nell'ambito del Piano d'azione.

In questo contesto il Codice prevede un ruolo importante dell'ANAC (Autorità nazionale anticorruzione ${ }^{12}$ ) che è incaricata di adottare bandi-tipo che contengono i criteri ambientali minimi al fine di agevolare l'attività delle stazioni appaltanti uniformandone le condotte (art. 71) e che, attraverso la sezione centrale del suo Osservatorio, deve monitorare sull'applicazione dei criteri ambientali minimi e sul raggiungimento degli obiettivi prefissati dal Piano d'azione per la sostenibilità dei consumi nel settore della pubblica amministrazione (art. 213, comma IX).

La pervasività dell'interesse ambientale nella disciplina codicistica emerge anche dalla lettura di disposizioni che, apparentemente, non sembrerebbero da ricondurre ad esso e che, invece,

\footnotetext{
${ }^{11}$ Oltre a ciò, il PAN fissava obiettivi per le Regioni e gli enti locali e stabilisce che almeno il $30 \%$ degli acquisti delle Regioni, delle Province, delle Città metropolitane e dei Comuni con oltre 15 mila abitanti adottino procedure di acquisto conformi ai criteri ambientali minimi (tale valutazione verrà realizzata rapportando il valore dei bandi sostenibili rispetto al totale dei bandi pubblicati). II nuovo Pan del 2013 ha aumenta la percentuale al $50 \%$ da realizzare entro il 2014, ma attualmente si è molto lontani da quelle previsioni programmatica assestandosi la spesa verde intorno al $10 \%$. Sul punto v. il Protocollo d'intesa tra il Ministero dell'Ambiente e della tutela del territorio e del mare e la Conferenza delle Regioni e delle Province autonome (17/134/CR08/C4-C5) del 2 agosto 2017 che, fra gli altri obiettivi, è volto alla diffusione e sensibilizzazione delle pubbliche amministrazioni all'applicazione dei CAM.

${ }^{12} \mathrm{M}$. RICCIARDO CALDERARO, La vigilanza e la regolazione nel Codice dei contratti pubblici: il controverso ruolo dell'ANAC, in C.E. GALLO, Autorità e consenso nei contratti pubblici, Dalle Direttive del 2014 al Codice del 2016, Torino, Giappichelli, 2017, 181; N. LONGOBARDI, L'Autorità Nazionale Anticorruzione e la nuova normativa sui contratti pubblici, in www.giustamm.it, 2016; G.M. RACCA, Dall'autorità sui contratti pubblici all'autorità nazionale anticorruzione: il cambiamento del sistema, in Dir. amm. 2015, 349.
} 
possono trovare un importante ambito di applicazione proprio nel contesto degli appalti verdi. II riferimento va alla nuova modalità di scelta del contraente di cui all'art. 65 del Codice: il partenariato per l'innovazione.

Si tratta di una procedura di scelta del contraente di tipo non ordinario basato su una stretta sinergia tra il soggetto pubblico e le imprese private: in ragione di ciò, è qualificato come un'espressione del partenariato pubblico privato ${ }^{13}$. Ricorrendo a questa procedura la pubblica amministrazione conta di sfruttare le potenzialità della ricerca da parte del settore privato, in quanto l'esigenza di acquisto del soggetto pubblico non è soddisfabile attraverso soluzioni già presenti sul mercato il quale, allora, viene sollecitato allo studio di soluzioni possibili. Ciò che si chiede al mercato è quindi di sviluppare prodotti, servizi o lavori innovativi ${ }^{14}$.

La possibilità, insita nella procedura in esame, di vari momenti di confronto tra l'amministrazione e più operatori economici richiede tuttavia l'adozione di cautele finalizzate alla tutela della par condicio e della riservatezza professionale, industriale e commerciale. Tra questi ${ }^{15}$, si noti che vige un divieto espresso di rivelare agli altri partecipanti informazioni riservate comunicate da un candidato o da un offerente che partecipa alle negoziazioni senza l'accordo di quest'ultimo.

\section{COSTI, PREZZI E OFFERTE PIÙ VANTAGGIOSE}

Una delle più importanti novità introdotte dal Codice del 2016 attiene ai criteri di aggiudicazione: sebbene ne vengano indicati due, il prezzo più basso e quello dell'offerta economicamente più vantaggiosa, il primo è da considerarsi del tutto residuale ${ }^{16}$.

Ai sensi dell'art. 95, comma II, le stazioni appaltanti, nel rispetto dei principi di trasparenza, di non discriminazione e di parità di trattamento, possono procedere all'aggiudicazione degli appalti sulla base del criterio dell'offerta economicamente più vantaggiosa "individuata sulla base del miglior rapporto qualità/prezzo" oppure sulla base dell'elemento prezzo o del costo "seguendo un criterio di comparazione costo/efficacia quale il costo del ciclo di vita" di cui all'art. 96.

Ai commi seguenti si stabilisce una triplice limitazione del criterio del prezzo più basso. Innanzitutto, sono individuati dei casi in cui è applicabile solo il criterio dell'offerta economicamente più vantaggiosa: ciò vale per i contratti relativi ai servizi sociali e di ristorazione ospedaliera, assistenziale e scolastica; i servizi ad alta intensità di manodopera (v. art. 50, comma I); i contratti relativi

\footnotetext{
${ }^{13}$ Sui molteplici significati dell'espressione sia consentito il rinvio a G. CREPALDI, Las formas del partenariado público-privado en el nuevo Código italiano de los contratos públicos y de las concesiones, in corso di pubblicazione sulla Rivista Contratación Administrativa Práctica.

${ }^{14}$ II partenariato per l'innovazione è disciplinato all'art. 65 del Codice dei contratti pubblici e delle concessioni che attua l'art. 31 della Direttiva europea 2014/24/UE. Si rinvia a S. FANTINI, II partenariato per l'innovazione, in Urb. e app., 2016, 955 ss.

${ }^{15}$ Alla stazione appaltante è imposto di informare tutti gli offerenti delle modifiche alle specifiche tecniche $\mathrm{o}$ ad altri documenti di gara diversi da quelli che stabiliscono i requisiti minimi; a seguito di tali modifiche, la stazione appaltante deve concedere agli offerenti un tempo sufficiente per modificare e ripresentare ove opportuno le offerte modificate.

${ }^{16}$ A tal proposito si noti che la Direttiva 2014/24/UE ne avrebbe consentito anche la totale abrogazione da parte della disciplina interna attuativa adottata dagli Stati membri.
} 
all'affidamento dei servizi di ingegneria e architettura e degli altri servizi di natura tecnica e intellettuale di importo pari o superiore a 40.000 euro $^{17}$.

Oltre a ciò, sono tassativamente stabiliti i casi in cui è in facoltà delle pubbliche amministrazioni ricorrere al criterio del minor prezzo: così per i lavori di importo pari o inferiore a 2.000.000 di euro, quando l'affidamento dei lavori avviene con procedure ordinarie, sulla base del progetto esecutivo; per i servizi e le forniture con caratteristiche standardizzate o le cui condizioni sono definite dal mercato; per i servizi e le forniture di importo fino a 40.000 euro, nonché per i servizi e le forniture di importo pari o superiore a 40.000 euro e sino alla soglia comunitaria solo se caratterizzati da elevata ripetitività, fatta eccezione per quelli di notevole contenuto tecnologico o che hanno un carattere innovativo.

Infine, si deve considerare che le stazioni appaltanti che dispongono l'aggiudicazione con il criterio del prezzo più basso, laddove ammesso, devono adeguatamente motivare tale scelta.

La possibilità di considerare e ponderare le qualità ambientali della prestazione quando il criterio prescelto è quello dell'offerta economicamente più vantaggiosa passa attraverso la determinazione di criteri oggettivi, fra i quali l'art. 95, comma VI, prevede le caratteristiche ambientali, il contenimento dei consumi energetici e delle risorse ambientali dell'opera o del prodotto, le caratteristiche innovative (lett. a); il possesso di un marchio di qualità ecologica dell'Unione europea (Ecolabel UE) in relazione ai beni o servizi oggetto del contratto, in misura pari o superiore al 30 per cento del valore delle forniture o prestazioni oggetto del contratto stesso (lett. b); il costo di utilizzazione e manutenzione avuto anche riguardo ai consumi di energia e delle risorse naturali, alle emissioni inquinanti e ai costi complessivi, inclusi quelli esterni e di mitigazione degli impatti dei cambiamenti climatici, riferiti all'intero ciclo di vita dell'opera, bene o servizio, con l'obiettivo strategico di un uso più efficiente delle risorse e di un'economia circolare che promuova ambiente e occupazione $\left(\right.$ lett. c) ${ }^{18}$; la compensazione delle emissioni di gas ad effetto serra associate alle attività dell'azienda calcolate secondo i metodi stabiliti in base alla raccomandazione n. 2013/179/UE della Commissione del 9 aprile 2013, relativa all'uso di metodologie comuni per misurare e comunicare le prestazioni ambientali nel corso del ciclo di vita dei prodotti e delle organizzazioni (lett. d).

In merito sono intervenute, in funzione attuativa, le Linee Guida dell'Autorità nazionale anticorruzione, n. 2 del 21 settembre 2016 (delibera ANAC n. 1005).

Si tenga conto che, proprio con l'obiettivo di valorizzare i profili qualitativi dell'offerta, il primo decreto correttivo al Codice dei contratti pubblici ha stabilito che il punteggio economico non può avere un peso superiore al trenta per cento (comma10 bis dell'art. 95).

Anche qualora il criterio di aggiudicazione fosse quello del prezzo più basso vi è la possibilità di considerare il profilo ambientale della prestazione ${ }^{19}$. L'amministrazione, infatti, può limitarsi a

\footnotetext{
${ }^{17}$ Sul punto v. P. PATRITO, II nuovo Codice dei contratti pubblici e il primo decreto correttivo (in corso di approvazione): primi spunti di riflessione, in Resp. civ. e prev., 2017, 335.

${ }^{18}$ Anche la Direttiva 18/2004/CE prevedeva la possibilità, sia pure più limitata, di includere i costi, come quello di utilizzazione, tra i criteri di valutazione dell'offerta economicamente più vantaggiosa. Allo stesso modo, la Commissione europea aveva più volte esortato a prendere in considerazione i costi ma ciò non si era tradotto in puntuale normativa interna.

${ }_{19}$ R. CAVALLO PERIN, G.M. RACCA, La cooperazione amministrativa europea nei contratti e servizi pubblici, in Riv. it. dir. pubbl. com., 2016, 1457.
} 
considerare l'aspetto economico valutando i costi: in altri termini, oltre a considerare il prezzo, valuterà i costi che la prestazione richiesta al mercato genera, come quelli sociali o ambientali.

Adottando un simile approccio, la stazione appaltante potrà preferire l'offerta che ha ad oggetto lavori, servizi o forniture che, nel corso della vita della prestazione, risultano meno costosi. Perciò, anche nel caso di aggiudicazione con il criterio del prezzo più basso, può assumere un ruolo centrale il costo del ciclo della vita (art. 95, II comma e art. 96).

\section{IL COSTO DEL CICLO DI VITA}

Il life cycle costing come determinazione fondamentale alla valutazione delle offerte sotto il profilo ambientale si è affermato attraverso la regolazione europea di soft law, volendo riferirsi a quegli atti mediante i quali le istituzioni comunitarie hanno orientato gli Stati membri verso la contrattazione verde, senza tuttavia poterli vincolare ad essa. In particolare si richiama la decisione n. 1600/2002/CE del 22 luglio 2002 laddove stabiliva, all'art. 3.6, che "è necessario promuovere una politica di appalti pubblici verdi che consenta di tener conto delle caratteristiche ambientali e di integrare eventualmente nelle procedure di appalto considerazioni inerenti al ciclo di vita" nonché, più specificamente, la Comunicazione della Commissione al Consiglio e al Parlamento Europeo "Politica Integrata dei Prodotti. Sviluppare il concetto di ciclo di vita ambientale", $\operatorname{COM}(2003) 302^{20}$.

Il costo del ciclo di vita è una formula riassuntiva che racchiude in sé molti e diversi costi accomunati dalla fonte: essi sono correlati ad un bene (opera, servizio, fornitura) e si producono durante il corso della sua vita. Nell'aggregato che costituisce il costo del ciclo di vita vi sono dunque ricompresi, in quanto pertinenti, tutti i costi che un prodotto, servizio o lavoro determina a partire dall'acquisizione delle materie e dei servizi necessari alla produzione, ai costi legati all'utilizzo, quali il consumo di energia e altre risorse; i costi di manutenzione; i costi relativi al fine vita, come i costi di raccolta, di smaltimento e di riciclaggio ${ }^{21}$.

A completamento del concetto del costo del ciclo della vita devono essere inclusi anche i costi imputati ad eventuali esternalità ambientali22 legate ai prodotti, servizi o lavori, purché il loro valore monetario possa essere determinato e verificato ${ }^{23}$.

\footnotetext{
${ }^{20}$ Tale Comunicazione ha assunto particolare importanza nell'affermazione del green public peocurement in Europa in quanto ha ufficialmente invitato gli Stati membri ad adottare un Piano d'Azione nazionale sul GPP entro I'anno 2006. In Italia il piano è stato adottato dal Ministero dell'Ambiente e della Tutela del Territorio e del Mare attraverso un ampio processo di consultazione con enti locali e parti interessate e con la collaborazione degli altri Ministeri Competenti (Economia e Finanze e Sviluppo Economico) e degli enti e strutture tecniche di supporto (CONSIP, ENEA, ISPRA, ARPA). Con molto ritardo, esso è stato adottato con decreto interministeriale l'11 aprile 2008 ed aggiornato il 10 aprile 2013.

${ }^{21}$ Si veda la definizione fornita all'art. 3, hhhh) che riconduce al "ciclo di vita" tutte le fasi consecutive o interconnesse, compresi la ricerca e lo sviluppo da realizzare, la produzione, gli scambi e le relative condizioni, il trasporto, l'utilizzazione e la manutenzione, della vita del prodotto o del lavoro o della prestazione del servizio, dall'acquisizione della materia prima o dalla generazione delle risorse fino allo smaltimento, allo smantellamento e alla fine del servizio o all'utilizzazione. Per un primo commento al concetto di life cycle costing introdotto dalle Direttive v. L. CARBONARA, Le nuove direttive sui contratti pubblici e la tutela dell'ambiente. I criteri di aggiudicazione, in www.giustamm.it, 2016.

${ }^{22}$ Per un chiarimento definitorio si rinvia alla disposizione-madre contenuta nel Considerando n. 96 della direttiva UE/24/2014: "Il concetto abbraccia i costi interni, come le ricerche da realizzare, lo sviluppo, la
} 
II life cycle costing, dunque, consente una valutazione più completa degli effettivi esborsi finanziari da sostenere per l'acquisizione, perché si considerano i costi direttamente sostenuti dalla pubblica amministrazione che acquista e quelli che vengono trasferiti su altri centri di spesa in quanto graveranno su bilanci futuri.

Non si negano, tuttavia, difficoltà applicative in merito alla uniforme quantificazione del costo aggregato. Per questa ragione si ritiene indispensabile elaborare metodologie comuni a livello dell'Unione europea per il calcolo dei costi del ciclo di vita per specifiche categorie di forniture 0 servizi; nel caso in cui tale metodo sia stato sviluppato, il suo utilizzo dovrebbe essere reso obbligatorio (v. già art. 68, par. 3 , della direttiva UE/24/2014) ${ }^{24}$.

Al fine di garantire che il life cycle costing non si traduca in uno strumento di possibile discriminazione ${ }^{25}$, le amministrazioni aggiudicatrici devono indicare nei documenti di gara la metodologia utilizzata per il calcolo dei costi del ciclo di vita, che deve soddisfare determinate condizioni: deve essere stata elaborata sulla scorta di informazioni scientifiche o sulla base di altri criteri oggettivi, verificabili e non discriminatori; deve essere accessibile a tutte le parti interessate; inoltre, i "dati richiesti devono poter essere forniti con ragionevole sforzo da operatori economici normalmente diligenti", compresi gli operatori economici di altri Stati membri, di paesi terzi parti dell'Accordo sugli appalti pubblici o di altri accordi internazionali che l'Unione è tenuta a rispettare o ratificati dall'Italia.

Con ciò, ancora una volta, il Codice dei contratti pubblici finisce per rivolgersi al settore privato richiedendo collaborazione al mercato, alle imprese che operano nel settore e che, meglio di qualunque soggetto pubblico, conoscono gli aspetti produttivi e quelli successivi relativi alla manutenzione, al mantenimento ed al fine vita ${ }^{26}$.

produzione, il trasporto, l'uso e la manutenzione e i costi di smaltimento finale ma può anche abbracciare costi imputabili a esternalità ambientali quali l'inquinamento causato dall'estrazione delle materie prime utilizzate nel prodotto ovvero causato dal prodotto stesso o dalla sua fabbricazione, a condizione che possano essere monetizzati e controllati”. Tali costi possono includere i costi delle emissioni di gas a effetto serra e di altre sostanze inquinanti, nonché altri costi legati all'attenuazione dei cambiamenti climatici.

${ }^{23}$ L'approccio richiama quello su cui si incentra il principio fondamentale del diritto ambientale "chi inquina paga". Sul punto si rinvia a C. VIVANI, Appalti sostenibili, Green public procurement e socially responsable public procurement, in Urb. e app., 2016, 993.

${ }^{24}$ L'allegato XIII al Codice contiene l'elenco degli atti legislativi dell'Unione e, ove necessario, degli atti delegati attuativi che approvano metodi comuni per la valutazione del costo del ciclo di vita. La Commissione può aggiornare tale elenco laddove una nuova normativa renda obbligatorio un metodo comune. Attualmente, I'allegato elenca la sola Direttiva 2009/33/CE del Parlamento europeo e del Consiglio recepita in Italia dal d.lgs. 3 marzo 2011, n. 24, che introduce norme per la promozione di veicoli a ridotto impatto ambientale e a basso consumo energetico nel trasporto su strada. Agli artt. 5 e 6 si prevede una metodologia di calcolo dei costi connessi al consumo energetico e ai costi relativi all'emissione di $\mathrm{CO} 2$ e alle emissioni di sostanze inquinanti che sono imputabili all'uso del veicolo al fine di quantificare il valore monetario.

${ }^{25}$ In funzione antidiscriminatoria, l'art. 96 afferma che se il metodo di calcolo del life cycle costing non è stato previsto per un'applicazione ripetuta o continua, non deve favorire né svantaggiare indebitamente taluni operatori economici.

${ }^{26}$ In senso critico v. L. DE PAULI, I "costi del ciclo di vita" nel nuovo codice degli appalti, in Urb. e app., 2016, 630 laddove ritiene che i costi e i dati che le imprese dovranno fornire, che si immaginano complessi, potrebbero essere forniti solo dalle imprese più grandi e strutturate a sfavore delle medio-piccole e micro imprese; il sistema finirebbe allora per impedire di fatto la partecipazione alle gare da parte di quelle situazioni dimensionali imprenditoriali che il Codice si propone, invece, di favorire. 
Per quanto si è detto, il sistema di selezione delle offerte basato sul life cycle costing è in divenire $^{27}$. La sua applicazione non può essere immediata perché richiede, con la collaborazione degli operatori economici, la definizione di metodologie comuni le quali, una volta definite, potranno e dovranno essere costantemente perfezionate.

\section{IL CICLO DI VITA COME CANONE DI PERTINENZA: CAUTELE}

La preoccupazione che attraverso la definizione e la valutazione del profilo ambientale dell'offerta possa ampliarsi indebitamente la discrezionalità della pubblica amministrazione è limitata da particolari cautele ${ }^{28}$.

La prima è una cautela generale con cui il legislatore esordisce già al primo comma dell'art. 95: i criteri di aggiudicazione non devono conferire alla stazione appaltante un potere di scelta illimitata dell'offerta; essi devono garantire la possibilità di una concorrenza effettiva e sono accompagnati da specifiche che consentono l'efficace verifica delle informazioni fornite dagli offerenti al fine di valutare il grado di soddisfacimento dei criteri di aggiudicazione delle offerte. E' dunque onere delle stazioni appaltanti l'accurata verifica delle informazioni e delle prove fornite dagli offerenti.

La seconda cautela risponde, prima che al diritto, alla ragione. Si tratta della regola della necessaria connessione del criterio con l'oggetto dell'appalto. Già con la pronuncia pilota della Corte di Giustizia Europea ${ }^{29}$, nota come sentenza Concordia Bus ${ }^{30}$, si stabilì che il principio della parità di trattamento non osta a che siano presi in considerazione nell'appalto criteri collegati alla tutela dell'ambiente, per il solo fatto che esistono poche imprese che hanno la possibilità di offrire un materiale che soddisfi i detti criteri ${ }^{31}$; ciò a condizione che i criteri, cumulativamente: siano collegati

\footnotetext{
${ }^{27}$ Le determinazioni richieste si basano sul LCA (Life Cycle Analysis) che ha lo scopo di fornire un elenco di tutti i profili di impatto ambientale del ciclo che parte dalla produzione, considera l'utilizzo e termina con lo smaltimento anche allo scopo di individuare possibili alternative. Già il sistema dell'Ecolabel, che è un'etichetta standardizzata che qualifica un prodotto come preferibile in termini di tutela dell'ambiente e che si riconosce ai soli prodotti e servizi il cui ciclo di vita sia integralmente caratterizzato da un ridotto impatto ambientale, si basa sulla LCA.

${ }^{28}$ In tema v. L. GILI, La nuova offerta economicamente più vantaggiosa e la discrezionalità amministrativa a più fasi, in Urb. e app., 2017, 24 ss.

${ }^{29}$ CGE, 17 settembre 2002, causa C-513/99, Comune di Helsinki - Concordia Bus, in Urb. e app., 2003, 168 con nota di M. BROCCA, Criteri ecologici nell'aggiudicazione degli appalti e in Foro amm.- CdS, 2002, 1936, con nota di M. LOTTINI, Appalti comunitari: sulla ammissibilità di criteri di aggiudicazione non prettamente economici.

${ }^{30} \mathrm{Nel}$ caso di specie il Comune di Helsinki aveva bandito una gara pubblica per la fornitura di autobus urbani e aveva individuato tra i criteri di valutazione per l'aggiudicazione della gara anche un criterio c.d. ecologico. La Società Concordia Bus, non risultata vincitrice della gara, ricorreva alla Corte di Giustizia Europea sollevando una questione di illegittimità del bando di gara che aveva previsto un punteggio particolarmente elevato per il criterio ecologico. La Corte di Giustizia Europea respingeva il ricorso affermando che "quando nell'ambito di un appalto pubblico relativo alla prestazione di servizi di trasporti urbani mediante autobus l'amministrazione aggiudicatrice decide di attribuire un appalto all'offerente che ha presentato l'offerta economicamente più vantaggiosa, può prendere in considerazione criteri ecologici, quali il livello di emissioni di ossido di azoto o il livello sonoro degli autobus, purché tali criteri siano collegati all'oggetto dell'appalto, non conferiscano all'amministrazione aggiudicatrice una libertà incondizionata di scelta, siano espressamente menzionati nel capitolato di appalto o nel bando di gara e rispettino tutti i principi fondamentali del diritto comunitario ed, in particolare, il principio di non discriminazione".

${ }^{31}$ Con una successiva pronuncia (4 dicembre 2003, causa C-448/01, EVN AG, Wienstrom GmbH contro Repubblica austriaca) la Corte di giustizia interviene in tema di energia elettrica ribadendo il principio affermato
} 
all'oggetto dell'appalto; non siano tali da conferire all'amministrazione aggiudicatrice una libertà incondizionata di scelta; siano espressamente menzionati nel capitolato d'appalto o nel bando di gara e rispettino tutti i principi fondamentali del diritto comunitario, in particolare il principio di non discriminazione ${ }^{32}$. Tali affermazioni sono state recepite, pressoché letteralmente, nelle Direttive 2004/17/CE e 2004/18/CE.

II Codice del 2016 interviene in merito spiegando che la relazione di connessione si può ritenere sussistente laddove i criteri riguardino lavori, forniture o servizi da fornire "sotto qualsiasi aspetto e in qualsiasi fase del loro ciclo di vita, compresi fattori coinvolti nel processo specifico di produzione, fornitura o scambio di questi lavori, forniture o servizi o in un processo specifico per una fase successiva del loro ciclo di vita, anche se questi fattori non sono parte del loro contenuto sostanziale" (art. 95, comma XI).

Quest'ultimo inciso consente di superare il collegamento tra il criterio di aggiudicazione e le caratteristiche sostanziali della prestazione che, invece, era stabilito dalla normativa comunitaria abrogata (v. Considerando n. 46 della Direttiva 2004/18/CE ${ }^{33}$ ) e che finiva per limitare la possibilità di valutare i profili ambientali della prestazione. Già la Corte di Giustizia europea aveva interpretato la disposizione nel senso di ammettere criteri di valutazione della prestazione non collegati ad una caratteristica intrinseca della stessa, ossia ad un elemento che si incorpori materialmente in essa ${ }^{34}$.

Questo passaggio è il presupposto di un'ulteriore evoluzione, operata dal Codice del 2016, che ricollega le specifiche tecniche e i criteri di valutazione delle offerte all'oggetto del contratto con precipuo riferimento all'intero del ciclo di vita dello stesso (artt. 68 e 95-96). Se, infatti, le une e gli altri

sul caso Concordia Bus e precisando che le amministrazioni aggiudicatrici, come possono scegliere liberamente i criteri di aggiudicazione dell'appalto, così possono anche stabilirne la ponderazione, purché ciò consenta una valutazione sintetica dei criteri adottati per individuare l'offerta economicamente più vantaggiosa (v. G. GARZIA, Bandi di gara per appalti pubblici e ammissibilità delle clausole c.d. ecologiche, in Foro amm., 2003, 3515; B. POGACE, I criteri ambientali negli appalti pubblici: dalle prime pronunce della corte di giustizia alla nuova direttiva 2004/18, in Urb. e App., 2004, 1385; A. GRATANI, L'energia elettrica da fonti rinnovabili e il vaglio del criterio di «positività ambientale» negli appalti, in Riv. giur. amb., 2004, 285; D.U. GALETTA. Vizi procedurali e vizi sostanziali al vaglio della Corte di giustizia, in Riv. it. dir. pub. com., 2004, 317; V. DE FALCO, L'utilizzo di fonti di energia rinnovabili come criterio di valutazione dell'offerta economicamente più vantaggiosa, in Dir. pub. comp. eur., 2004, 889.

32 A questo scopo, si deve sottolineare l'importanza del canone della proporzionalità: le condizioni ecologiche inserite nella documentazione di gara sotto i vari profili che già si sono esaminati, non possono tradursi in condizioni eccessivamente onerose per gli operatori economici che partecipano alla gara. Così sarebbe se si prevedessero delle certificazioni ambientali molto costose, in relazione al valore del contratto, da rendere non conveniente la partecipazione alla gara. Sul principio di proporzionalità si rinvia a S. COGNETTI, Principio di proporzionalità, Torino, Giappichelli, 2011, 10 ss.; S. VILLAMENA, Contributo in tema di proporzionalità amministrativa. Ordinamento comunitario, italiano e inglese, Milano, Giuffrè, 2008, 7 ss.

33 Del seguente tenore letterale: "la determinazione di tali criteri dipende dall'oggetto dell'appalto in quanto essi devono consentire di valutare il livello di prestazione che ciascuna offerta presenta rispetto all'oggetto dell'appalto, quale definito nelle specifiche tecniche, nonché di misurare il rapporto qualità/prezzo di ciascuna offerta". Con ciò, si stabiliva un sistema basato su una duplice correlazione: le specifiche tecniche dovevano essere riferite al contenuto materiale dell'appalto e i criteri di valutazione dovevano essere determinati in funzione della valutazione proprio degli aspetti materiali della prestazione.

${ }^{34}$ CGE, 10 maggio 2012, in causa C-368/10, Commissione c. Paesi Bassi (in Urb. e app., 2012, 1135, con nota di R. CARANTA, L'incerta sostenibilità degli appalti pubblici) nota come sentenza sul caso Max Havelaar, ha ritenuto non necessario che il criterio ecologico implichi un effetto sulle caratteristiche intrinseche del prodotto ben potendo attenere solo al processo di produzione. Con ciò la Corte ha ritenuto legittimo che la pubblica amministrazione possa stabilire criteri che preferiscono prodotti derivanti dal commercio equo e solidale.

Revista do Direito [ISSN 1982-9957]. Santa Cruz do Sul, v. 1, n. 54, p. 86-98, jan./abr. 2018. https://online.unisc.br/seer/index.php/direito/index 
possono riferirsi a "tutte le fasi consecutive e/o interconnesse compresi la ricerca e lo sviluppo da realizzare, la produzione, gli scambi e le relative condizioni, il trasporto, l'utilizzazione e la manutenzione, della vita del prodotto o del lavoro o della prestazione del servizio, dall'acquisizione della materia prima o dalla generazione delle risorse fino allo smaltimento, allo smantellamento e alla fine del servizio o all'utilizzazione", in base a quanto stabilisce l'art. 3, I comma, lett. hhhh), allora, non vi sono più collegamenti necessitati al contenuto materiale del servizio, dell'opera o della fornitura e l'oggetto del contratto viene a sovrapporsi al ciclo di vita di tale prestazione.

Questa nuova e più ampia impostazione è il risultato di una precisa volontà legislativa di valorizzare il profilo ambientale e sociale della prestazione, come espressamente affermato al Considerando n. 97 della Direttiva 24/2014/UE.

La medesima ratio è rintracciabile nella previsione che, in modo innovativo rispetto al sistema previgente, ammette la competizione solo in relazione ai criteri qualitativi, nel senso che fermo il prezzo o il costo fissato dalla stazione appaltante, le imprese si troverebbero a concorrere esclusivamente in base alla qualità e valutate con parametri non economici. Questo nuovo criterio di aggiudicazione presenta il vantaggio di impedire ribassi anomali che inciderebbero sulla qualità, anche ambientale della prestazione ${ }^{35}$.

L'ultima cautela a limitazione della discrezionalità dell'amministrazione, contro contegni arbitrari lesivi della concorrenza, è da ravvisarsi nel rispetto del principio di trasparenza e nell'adempimento agli obblighi di pubblicità: le modalità di calcolo dei costi devono essere accessibili da parte di tutti. II Codice impone che esse siano inserite nella documentazione di gara e richiede che gli offerenti possano conoscere sin dall'inizio della gara i dati che saranno oggetto di valutazione, la procedura ed i parametri. Ciò è determinate ai fini della partecipazione e della presentazione di un'offerta congrua su misura.

\section{MEDIE-PICCOLE-MICRO IMPRESE, PRODUZIONI CHILOMETRI ZERO E FILIERA CORTA}

Nella più recente normativa codicistica il legislatore strumentalizza in modo rilevante la regolazione sulla contrattualistica pubblica per la tutela di situazioni imprenditoriali e professionali che richiedono di essere promosse e valorizzate, sulla base degli indirizzi "unionali"36.

Dell'imprenditoria più debole di fronte al circuito delle commesse pubbliche il Codice si occupa in svariate occasioni ${ }^{37}$ : così, l'art. 30 , comma VII richiede che i criteri di partecipazione alle gare debbano essere tali da non escludere le micro, piccole e medie imprese e l'art. 51, allo scopo di

\footnotetext{
${ }^{35}$ Perplessità sono manifestate da C. LAMBERTI e S. VILLAMENA, Nuove direttive appalti: "sistemi di selezione" e "criteri di aggiudicazione", in Urb. e app., 2015, 882 che ritengono particolarmente complicata la determinazione del prezzo da parte delle stazioni appaltanti.

${ }^{36}$ Trattasi di un neologismo che talora si utilizza per sostituire l'aggettivo "comunitario" da quando alla Comunità europea si è sostituita l'Unione europea.

${ }^{37}$ Si tratta delle imprese definite all'art. 3, lett. aa): microimprese, piccole e medie imprese», le imprese come definite nella Raccomandazione n. 2003/361/CE della Commissione del 6 maggio 2003. In particolare, sono medie imprese le imprese che hanno meno di 250 occupati e un fatturato annuo non superiore a 50 milioni di euro, oppure un totale di bilancio annuo non superiore a 43 milioni di euro; sono piccole imprese le imprese che hanno meno di 50 occupati e un fatturato annuo oppure un totale di bilancio annuo non superiore a 10 milioni di euro; sono microimprese le imprese che hanno meno di 10 occupati e un fatturato annuo oppure un totale di bilancio annuo non superiore a 2 milioni di euro.
} 
favorirle, prevede la suddivisione dell'oggetto del contratto in lotti; si stabilisce che i contratti sottosoglia ${ }^{38}$ debbano essere affidati nel rispetto del principio di rotazione degli inviti e degli affidamenti e in modo da assicurare l'effettiva possibilità di partecipazione delle micro, piccole e medie imprese e che l'aggregazione degli acquisti attraverso la creazione di un sistema di reti di committenza garantisca la partecipazione delle stesse; ancora, è affidata alla Cabina di regia sugli appalti pubblici39 e all'ANAC l'analisi dei dati sull'efficientamento del sistema di acquisto attraverso le centrali di committenza, nell'ambito dei quali devono essere evidenziati anche gli accorgimenti adottati per garantire l'effettiva partecipazione delle micro, piccole e medie imprese. Infine, l'art. 83 prevede a favore di queste imprenditoriali minori che l"offerta non debba essere corredata, a pena di esclusione, dall'impegno di un fideiussore qualora l'offerente risultasse affidatario.

Accanto a questo interesse, se ne affianca un altro correlato: quello di favorire le situazioni imprenditoriali più limitate operanti sul territorio in grado di offrire prodotti nella medesima zona, in questo modo contenendo anche le problematiche ambientali legate al trasporto delle merci. Si riconosce, infatti, la possibilità di introdurre nella valutazione dell'offerta dei criteri premiali per agevolare la partecipazione alle procedure di affidamento per le micro, piccole e medie imprese, per i giovani professionisti e per le imprese di nuova costituzione e si prevede di un maggior punteggio relativo all'offerta concernente beni, lavori o servizi che "presentano un minore impatto sulla salute e sull'ambiente ivi inclusi i beni o prodotti da filiera corta o a chilometro zero"40.

Si tratta di un lessico nuovo ${ }^{41}$ mutuato dalla regolazione comunitaria, che il legislatore utilizza per introdurre criteri di qualità specifica: la qualità ambientale. In tal senso, si veda l'art. 144 inerente i servizi di ristorazione il cui affidamento richiede la valutazione dell'offerta tecnica tenendo conto di fattori come la qualità dei generi alimentari con particolare riferimento a quella di prodotti biologici, tipici e tradizionali, di quelli a denominazione protetta, nonché di quelli provenienti da sistemi di filiera corta e da operatori dell'agricoltura sociale, il rispetto delle disposizioni ambientali in materia di green economy, dei criteri ambientali minimi pertinenti e della qualità della formazione degli operatori.

\section{OSSERVAZIONI CONCLUSIVE SULLA QUALIFICAZIONE AMBIENTALE DELLE STAZIONI APPALTANTI (E DEGLI ENTI)}

Per quanto si è detto, è possibile ritenere che la nuova regolazione del Codice dei contratti pubblici sia certamente in grado di offrire una tutela più efficace, in quanto trasversale, dei valori ambientali, da intendersi in senso ampio ${ }^{42}$.

\footnotetext{
${ }^{38}$ Trattasi di contratti di minor valore in base alle soglie determinate dall'art. 35 (così, ad esempio, gli affidamenti di lavori per importi inferiori a Euro 5.225.000).

${ }^{39}$ Istituita presso la Presidenza del Consiglio dei ministri, la Cabina di regia ha il compito, fra gli altri, di compiere ricognizioni sullo stato di attuazione del Codice e di proporre eventuali soluzioni correttive e di miglioramento, nonché di segnalare specifiche violazioni all'ANAC per gli interventi di competenza. In merito disciplina della Cabina di Regia si rinvia a quanto è previsto all'art. 212, rubricato Indirizzo e coordinamento.

${ }^{40}$ Ciò, naturalmente, deve avvenire compatibilmente con il diritto dell'Unione europea e con i principi di parità di trattamento, non discriminazione, trasparenza e proporzionalità.

41 S. VILLAMENA, Codice dei contratti pubblici 2016. Nuovo lessico ambientale, clausole ecologiche, sostenibilità, economicità, in Riv. giur. edilizia, 2017, 101 ss.

${ }^{42} \mathrm{Si}$ veda l'art. 23 che nell'occuparsi della progettazione in materia di lavori pubblici afferma che è intesa ad assicurare la conformità alle norme ambientali, urbanistiche e di tutela dei beni culturali e paesaggistici (lett. c),
} 
La scelta di non racchiudere la regolazione dei c.d. contratti verdi in un corpus di norme dedicate all'interno del Codice ha favorito l'inclusione della considerazione ambientale ad ogni occasione possibile, in relazione cioè alla disciplina di ogni determinazione che possa incidere sullo scopo di favorire l'acquisto pubblico eco-sostenibile: dalla redazione del progetto di fattibilità tecnicoeconomica, ai requisiti di partecipazione, alle certificazioni ambientali relative il processo produttivo e la prestazione, alle modalità di scelta del contraente, ai criteri di valutazione dell'offerta, finanche alle clausole di esecuzione del contratto.

Si tratta, infatti, di una regolazione complessivamente orientata agli acquisti eco-sostenibili, caratterizzata da una più marcata flessibilità ${ }^{43}$, funzionale a perseguire gli obiettivi di qualità ambientale sempre nel contesto dei principi comunitari, primo fra tutti l'imparzialità. Tale approccio normativo, aperto e dinamico, dovrebbe garantire il rapido adeguamento ed un continuo perfezionamento delle misure.

Non si tratta, tuttavia, di un punto di arrivo. Occorrerà innanzitutto verificare il grado di effettività della nuova regolazione, vigilando sulla capacità delle amministrazioni di dare la massima applicazione alla normativa, sfruttandone le potenzialità.

Per questa ragione, uno degli aspetti su cui occorrerebbe investire è quello della qualificazione ambientale delle stazioni appaltanti nel contesto del nuovo sistema di valutazione di cui all'art. 38 del Codice dei contratti pubblici4 ${ }^{44}$. Tale disposizione ha introdotto un'importante novità, prevedendo la costituzione presso l'ANAC di un apposito elenco delle stazioni appaltanti qualificate in base a determinati parametri (ambiti di attività, bacini territoriali, tipologia, complessità del contratto, fasce d'importo) e ai requisiti tecnico-organizzativi in merito ai quali si deve attendere la determinazione con Linee guida ${ }^{45}$.

E' proprio nell'ambito della definizione dei requisiti utili alla qualificazione che occorre valorizzare quelli ambientali fra quelli "premianti"46. L'art. 38, IV comma, lett. b), già lo prevede: la disposizione individua, infatti, cinque ipotesi di premialità fra cui l'applicazione di criteri di sostenibilità

un limitato consumo del suolo (lett. d), il rispetto dei vincoli idrogeologici, sismici e forestali nonché degli altri vincoli esistenti (lett. e), il risparmio e l'efficientamento energetico nonché il recupero energetico nella realizzazione e nella successiva vita dell'opera; anche in questo caso si fa riferimento alla valutazione del ciclo di vita e della manutenibilità delle opere, alla compatibilità con le preesistenze archeologiche e alla compatibilità geologica, geomorfologica, idrogeologica dell'opera.

43 La precedente regolazione codicistica, invece, non consentiva all'amministrazione di arricchire le proprie conoscenze ambientali attraverso l'apprendimento e il dialogo con gli operatori economici, rilevandosi in ciò un grave limite. Sul punto v. G. FIDONE, Gli appalti verdi all'alba delle nuove direttive: verso modelli più flessibili orientati a scelte eco-efficienti, in Riv. it. dir. pubbl. com., 2012, 819 ss.

${ }^{44}$ Su tale sistema si rinvia a C. LAMBERTI, La qualificazione delle stazioni appaltanti, in Urb. e app., 2016, 1293.

${ }^{45}$ Si noti che l'art. 216, comma 10, dispone che "Fino alla data di entrata in vigore del sistema di qualificazione delle stazioni appaltanti di cui all'articolo 38, i requisiti di qualificazione sono soddisfatti mediante l'i-scrizione all'anagrafe di cui all'articolo 33-ter del decreto legge 18 ottobre 2012, n. 179, convertito, con modificazioni, dalla legge 17 dicembre 2012, n. 221". In ragione di ciò, poiché è neces-saria l'adozione di disposizioni attuative, al momento non ancora adottate, tutte le stazioni appaltanti devono ritenersi qualificate me-diante la semplice iscrizione presso l'ANAC.

${ }^{46}$ La disposizione individua, innanzitutto, dei requisiti "di base" quali, fra gli altri, la stabilità delle strutture organizzative stabili; la presenza di dipendenti aventi specifiche competenze in rapporto alle attività; l'allestimento di un sistema di formazione ed aggiornamento del personale; il numero di gare svolte nel quinquennio; il rispetto dei tempi previsti per i pagamenti di imprese fornitrici. 
ambientale e sociale nell'attività di progettazione e di affidamento ${ }^{47}$. La rilevanza e l'effettivo peso che tale aspetto potrà assumere nella valutazione dipenderà dalla normazione attuativa dell'ANAC, in via di approvazione.

Si tenga conto che alla qualificazione delle sta $\neg$ zioni appaltanti sono ricollegati degli incentivi: la valutazione positiva della stazione appaltante viene comunicata dall'ANAC all'amministrazione di appartenenza perché ne tenga conto ai fini della valutazione della performance organizzativa e gestionale dei dipendenti in-teressati. Inoltre, una quota degli introiti determinati dalle sanzioni derivanti dall'attività di vigilanza svolta dall'ANAC dovrà essere destinata all'amministrazione della stazione appaltante affinché venga destinata a remunerare il risultato dei dirigenti e dei dipendenti appartenenti alle unità organizzative competenti per i proce $\neg$ dimenti d'appalto (comma IX).

Infine, si può considerare che un sistema di approvvigionamento improntato all'ecosostenibilità può essere valutato anche a favore dell'ente, nel contesto di un differente sistema di qualificazione.

I benefici del green public procurement, infatti, si misurano anche in termini di miglioramento di immagine dell'ente che convertendo il proprio sistema di approvvigionamento offre un esempio di consapevolezza e responsabilità ambientale ai cittadini e agli altri enti. Questo beneficio, che si può considerare un'esternalità positiva dell'acquisto verde, assume rilevanza nell'ambito del processo di valutazione ambientale degli enti che si sta affermando i molti paesi europei. E' un fenomeno che riguarda principalmente gli enti territoriali che, come le imprese, richiedono agli organismi internazionali o nazionali di certificazione di valutare la propria attività (normativa, programmatoria, di gestione e controllo) alla luce di criteri e targets ambientali ${ }^{48}$.

Ciò consente all'ente di possedere una approfondita e documentata conoscenza dei propri comportamenti in ambito ambientale, di monitorare lo sviluppo delle procedure rilevando carenze ed errori in funzione del continuo miglioramento e perfezionamento delle prestazioni.

\section{COMO CITAR ESSE DOCUMENTO:}

CREPALDI, Gabriella. Gli acquisti eco-sostenibili nel nuovo Codice dei contratti pubblici italiano: considerazioni sul life cycle costing. Revista do Direito, Santa Cruz do Sul, v. 1, n. 54, p. 86-98, jun. 2018. ISSN 1982-9957. Disponível em: <https://online.unisc.br/seer/index.php/direito/article/view/12046>. Acesso em: doi:http://dx.doi.org/10.17058/rdunisc.v1i54.12046.

\footnotetext{
${ }^{47}$ Le altre situazioni premiali sono da ricollegare alla valutazione positiva dell'ANAC in ordine all'attuazione di misure di prevenzione dei rischi di corruzione e promozione della legalità; alla presenza di sistemi di gestione della qualità conformi alla norma UNI EN ISO 9001, certificati da or-ganismi accreditati; alla disponibilità di tecnologie telematiche nella gestione di procedure di gara; al livello di soccombenza nel contenzioso; infine, viene considerata

${ }^{48}$ In Italia, la prima certificazione ambientale ISO 14001 è stata rilasciata nel 1999 al Comune di Varese Ligure. Oggi sono migliaia gli enti locali italiani dotati della certificazione ISO 14001 o EMAS.
} 